\title{
Fundamentals of silicon photonics: a course for upperclassmen in optical engineering
}

\section{Azad Siahmakoun}

Azad Siahmakoun, "Fundamentals of silicon photonics: a course for upperclassmen in optical engineering ," Proc. SPIE 10741, Optics Education and Outreach V, 1074103 (14 September 2018); doi: 10.1117/12.2320369

EDent: SPIE Optical Engineering + Applications, 2018, San Diego, California, United States 


\title{
Fundamentals of Silicon Photonics: Design, Fabrication, and Testing A Course for Senior Optical Engineering Majors
}

\author{
Azad Siahmakoun \\ Department of Physics and Optical Engineering \\ Rose-Hulman Institute Of Technology \\ Terre Haute, Indiana USA 47803 \\ siahmako@rose-hulman.edu
}

\begin{abstract}
This is an introductory course in silicon photonics for senior and graduate students. Topics covered in this course include: basic properties of silicon waveguides; the essentials of SOI waveguide fabrication; dispersion and propagation loss in Si waveguides; analysis and design of various passive silicon devices; modeling and characterization of silicon modulators; integrated photonic circuit; and layouts for E-beam lithography. This course also includes a laboratory to enhance student's learning. Students fabricate a thermo-optic MZI modulator in the cleanroom and then characterize its performance. We will discuss experimental work by student groups and comment on student's learning in this class.
\end{abstract}

Keywords: Silicon Photonics, Optical Engineering, Introductory Course SOI Waveguide Thermo-Optic MZI Modulator

\section{INTRODUCTION}

The Learning objectives for this introductory course in silicon-photonics include: basic properties of silicon waveguides; the essentials of silicon-on-insulator (SOI) waveguide fabrication (WGs); dispersion and propagation loss in Si WGs; analysis and design of various passive silicon devices such as strip and rib waveguides, directional couplers, Y-branch splitter/combiner, Mach-Zehnder interferometer; design and modeling of Bragg grating for light coupling in and out of devices/circuits; develop matrix method analysis in order to determine the transfer function of a passive device; modeling and characterization a MachZehnder silicon modulator (MZI); use free shareware KLayout to generate integrated photonic circuit (PIC) layouts for E-beam lithography.

This is a research based learning class for senior and graduate students on silicon-photonics ( $\mathrm{SiPh})$ design, fabrication, and testing where they are given both theoretical and laboratory projects to do. Students will be using various software in this course: MATLAB, KLayout, Excel, and Lumerical.

We have also obtained educational license agreement with Lumerical Company through their Knowledge Exchange program for the free use of their Mode Solutions and Interconnect software. Students use Lumerical in order to evaluate mode propagation in various Si-WGs and passive components as well as to model and evaluate performance characteristics of their designed PICs. The textbook for the course is the one by Lukas Chrostowski and Michael Hochberg, titled: "Silicon Photonics Design from Devices to Systems." This textbook includes many useful codes and examples developed in Lumerical as well as MATLAB [1].

Projects assigned to each student is to design and model a passive or an active device such as ring resonator filters or Mach-Zehnder Interferometers with applications in either sensing or communications. For example the MZI device must be consisted of waveguides, couplers, Y-splitters, and input/output Bragg gratings. These devices are to operate at $1550 \mathrm{~nm}$.

Optics Education and Outreach V, edited by G. Groot Gregory, Proc. of SPIE Vol. 10741, 1074103

(C) 2018 SPIE · CCC code: 0277-786X/18/\$18 · doi: 10.1117/12.2320369 
This course also has a fabrication and testing laboratory to enhance student's learning with this technology. The goal is to make a light modulator on an SOI wafer and characterize its performance using a singlemode fiber-optic link. An example process flow for fabricating this device is provided in Table 1.

\section{Modeling a Device}

The MZI is an optical device that uses the Interferometer effect: an input signal is divided between two paths; a given phase change is applied to the signals in each path; and finally, both signals are recombined at the output (see Figure 1).

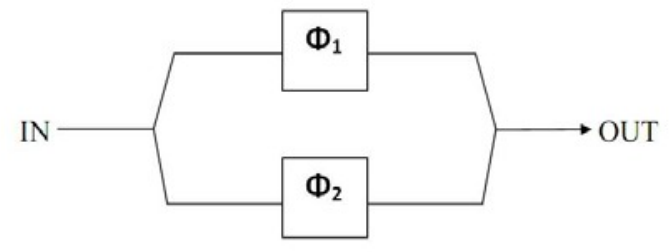

Figure 1: Mach Zehnder Interferometer (MZI) scheme.

Where $\Phi_{1}$ and $\Phi_{2}$ are the phase changes for each optical path. If we analyze the interferometer as a system, it is possible to find its transfer function equation:

$$
H_{12}=\cos ^{2}(\Delta \varphi)=\cos ^{2}\left(\frac{2 \pi}{\lambda}\left(n_{2} l_{2}-n_{1} l_{1}\right)\right)
$$

Where $\Delta \varphi=\frac{2 \pi}{\lambda} \Delta n_{e f f} \Delta \mathrm{L}$ is the phase difference between the branches and $\Delta n_{e f f}$ is the variation of the effective index. It is necessary to note that this transfer function works in an ideal case, where system losses and anisotropies are not considered. However, the refractive index $n$ can be changed by injection of charge carriers (Charge-Carrier Effects), as shown in the following equations.

$$
\begin{gathered}
\Delta n=-\left(\frac{e^{2} \lambda^{2}}{8 \pi^{2} c^{2} \varepsilon_{0} n}\right)\left[\frac{\Delta N_{e}}{m_{c e}^{*}}+\frac{\Delta N_{h}}{m_{c h}^{*}}\right] \\
\Delta k=\left(\frac{e^{3} \lambda^{2}}{4 \pi^{2} c^{2} \varepsilon_{0} n}\right)\left[\frac{\Delta N_{e}}{m_{c e}^{* 2} \mu_{e}}+\frac{\Delta N_{h}}{m_{c h}^{* 2} \mu_{h}}\right]
\end{gathered}
$$

Where $\Delta N_{e}$ and $\Delta N_{h}$ are the charge density for electrons and holes, $e$ is the electron charge, $\varepsilon_{0}$ is the permittivity of free space, $m_{c e}^{*}$ and $m_{c h}^{*}$ are the effective mass of electrons and holes, $\mu_{e}$ and $\mu_{h}$ are the mobility of the electron and hole, respectively.

If we use thermic control heaters instead of P-N junctions, it is possible to achieve differences in the refractive index of $\sim 10^{-2}$. In this case, the physics behind the problem is much simpler if we ignore the effects of thermal displacement and expansion. The simplest model for a change in the refractive index is given by the equation:

$$
n=n_{0}+\frac{d n}{d T} \Delta T
$$

Where $n_{0}$ is the refractive index of the material, $\frac{d n}{d T}$ is the thermo-optic coefficient and $\Delta T$ is the temperature difference between two points. Since the thermo-optic coefficient is different for each material, the factor hat we need to analyze will be $\frac{d n_{e f f}}{d T}$. These values can be obtained numerically. 
These two types of modulation behave in different ways, and in practice, switching frequencies of the order of $\mathrm{MHz}$ have been achieved with Thermo-optic MZM [2], while frequencies of $\sim 10 \mathrm{GHz}$ have been achieved with PIN MZM [3].

Figure 2 shows the numerically calculated results of temperature dependence of the effective index, this simulation is made ignoring dispersion effects or any loss by Joule effect. These values are calculated for 3 geometries $220 \times 500 \mathrm{~nm}$ strip waveguide (black), 220×500 nm with $90 \mathrm{~nm}$ rib waveguide and the multimodal $220 \times 2500 \mathrm{~nm}$ strip waveguide used in this design (blue).

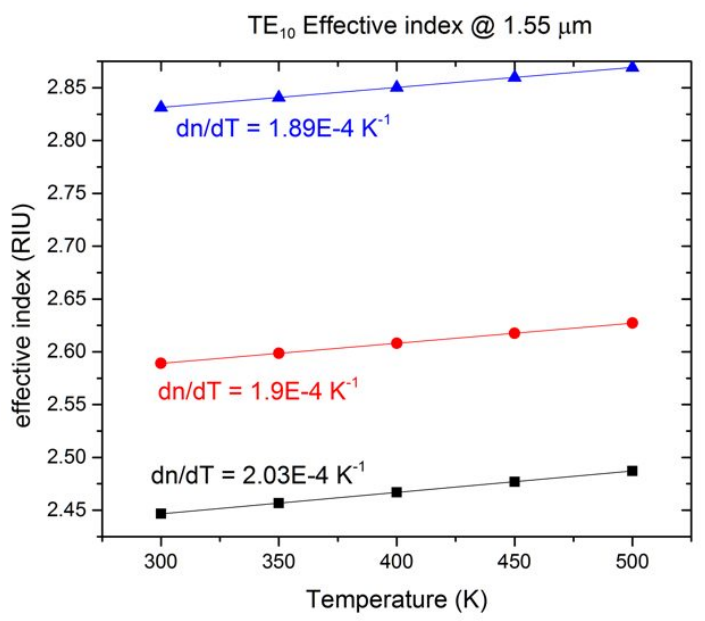

Figure 2: Effective index dependence on temperature for the TE10 mode on different waveguides.

Figure 3 shows the calculated values of $\Delta n$ as a function of the charge density, it's clear here that using $\mathrm{P}^{+}$ doped zones are more useful in a design since the plasma effect is more pronounce for the lower density values.

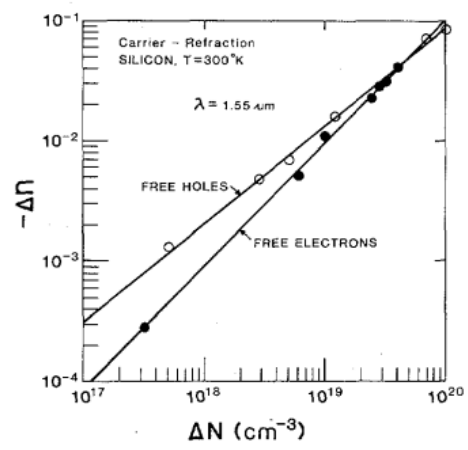

Figure 3: Refractive index dependence with concentration of impurities.

\section{EXPERIMENTATION}

The first part is to fabricate an SOI wafer using PECVD and dry oxidation processes in the cleanroom. The modulator devices are designed to work with a strip waveguide $220 \times 500 \mathrm{~nm}$. This fabrication process is a 3 mask process with each MZI to have seven different kinds of modulation schemes to choose from. This includes active zone with P-N junctions (4 variations), with or without active zone with heaters. Fabrication steps include photolithography, etching WGs, doping process to create PN-junction, depositing oxide cladding, and making the metal contacts. 
Table 1: Process flow for fabricating an SOI based thermos-optic MZI.

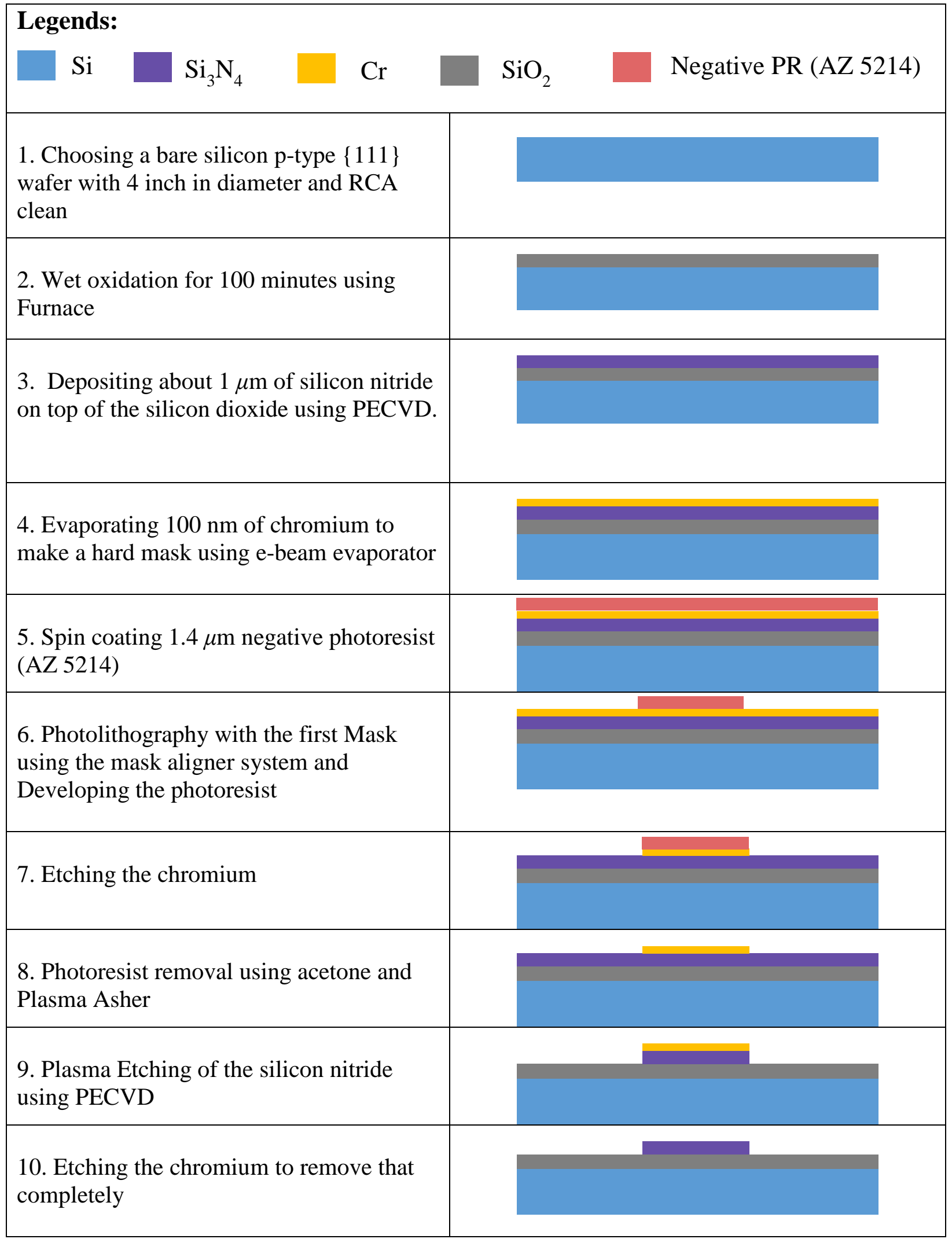


The following is the procedure for fabrication process using an SOI wafer. The designed mask has three types of patterns:

- 1 balanced MZI Normally Closed switch (NC).

- 1 unbalanced NC MZI.

- 1 unbalanced Normally Open (NO) MZI.

All of MZI layouts are based on two Y-Junctions with an angle of $18^{\circ}$. These devices are designed to work with a strip waveguide $220 \times 500 \mathrm{~nm}$. Figure 4 shows the waveguide cross-section and Fig.5 shows the footprints of each MZI with its dimensions.

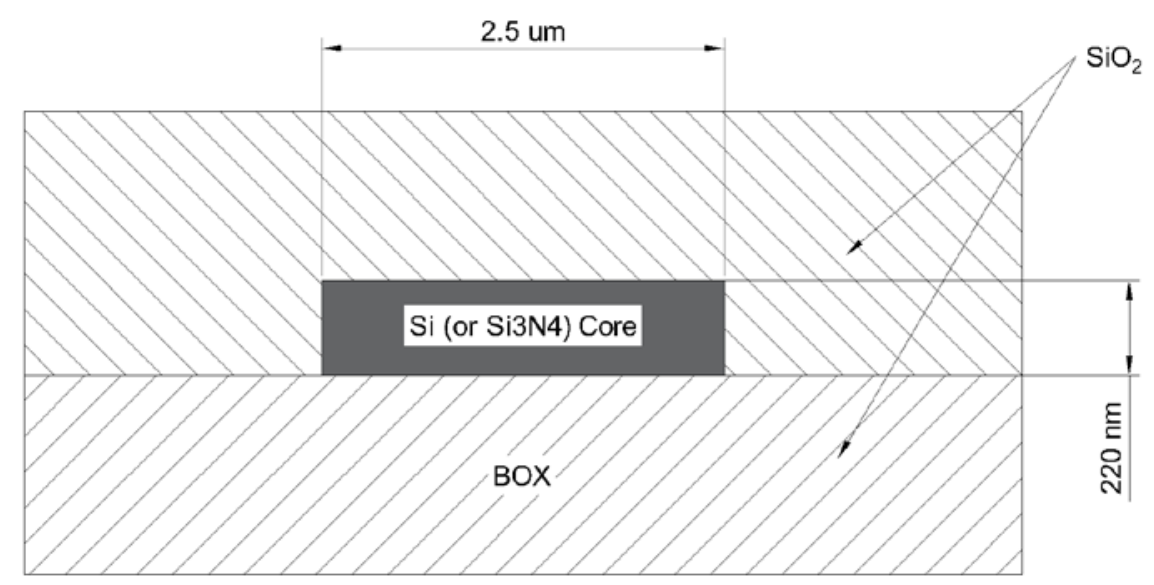

Figure 4: Waveguide cross-section and dimensions

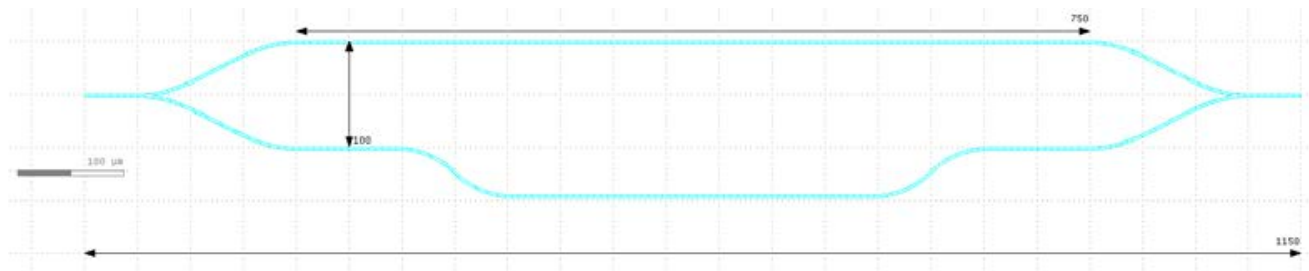

Figure 5: MZI principal dimensions.

There are 7 different kinds of MZI modulation schemes available to use:

- Active zone with P-N junctions:

o Single drive large P-N slab on one branch.

o Dual drive balanced P-N slab on two branches (Common node for the two branches).

o Single drive P-N dots array.

o Dual drive P-N dots array.

- Active zone with heaters:

o Single Drive Heater.

o Dual Drive Heater.

- Mixed Active zone:

o One branch with heaters the other with P-N dots array. 
Figure 6 shows the different types of modulation schemes. In the layout, the devices are arrange as Units Cells (UC). Each UC has 4 Inputs and 4 outputs and a size of $7 \times 6 \mathrm{~mm}$. In one UC are included the 7 kinds of MZI, each input illuminate 2 devices at the same time, the outputs are aligned to measure from 1 device at a time independent of the source. Figure 7 shows the footprint of a UC.

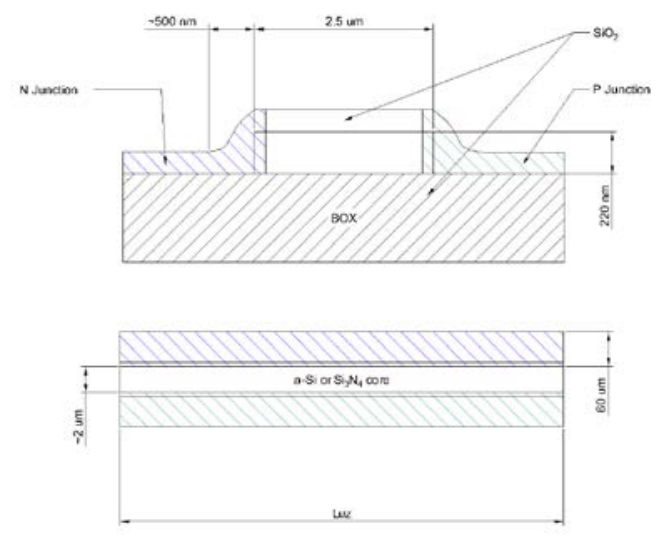

(a)
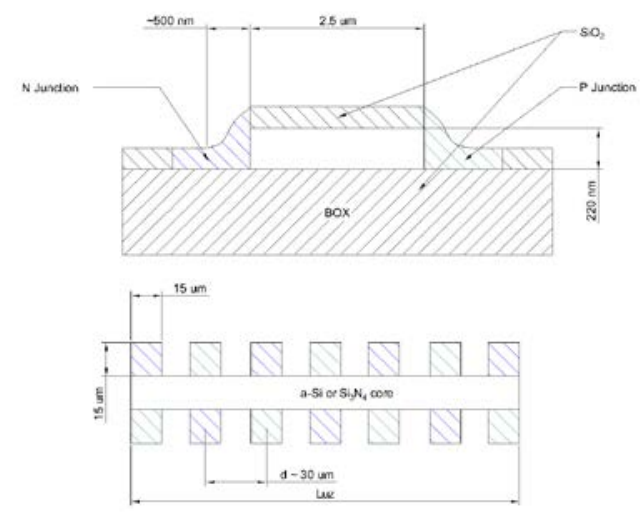

(b)

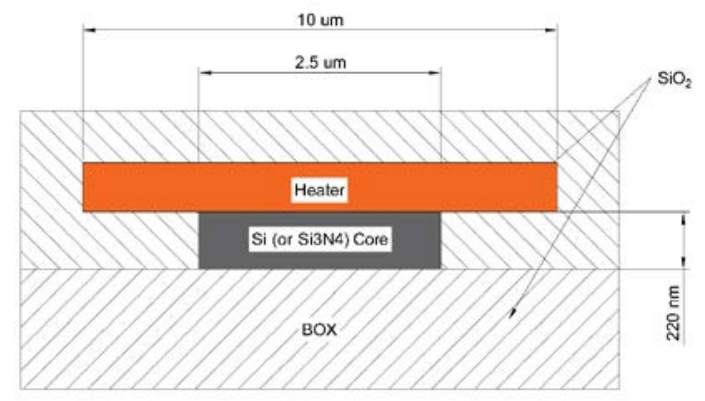

(c)

Figure 6: Types of modulation schemes implemented on the mask designed. (a) and (b) PiN junction based, (c) thermo-optic based.

As the mask for $\mathrm{P}$ junctions is the same for $\mathrm{N}$ junctions (a rotational symmetry was considered for the design), the distribution on the wafer is mirrored around the $\mathrm{Y}$ axis, the schematic arrangement is showed in figure 8 .

In order to process a wafer, the following steps are carried out (the RCA clean steps are neglected from the list):

1. Select the type of MZI to use. (Balanced NO, Unbalanced NO, Unbalanced NC).

2. Select the type of modulation.

3. Select an UC from the wafer, each UC has an align mark to facilitate the separation between UC's.

4. Prepare the wafer for oxidation process (deep clean).

5. BOX layer- Thermal oxidation is used to achieve better uniformity than the PECVD film.

6. Annealing process to relief stress on wafer (optional).

7. Core layer- Use the PECVD machine software with the recipe $\alpha$-Si for $250 \mathrm{~nm}$ thickness. 
8. For nitride core material, an annealing process it's recommended after the deposition. The annealing time must not be longer than 30 minutes and the temperature not higher than $700{ }^{\circ} \mathrm{C}$.

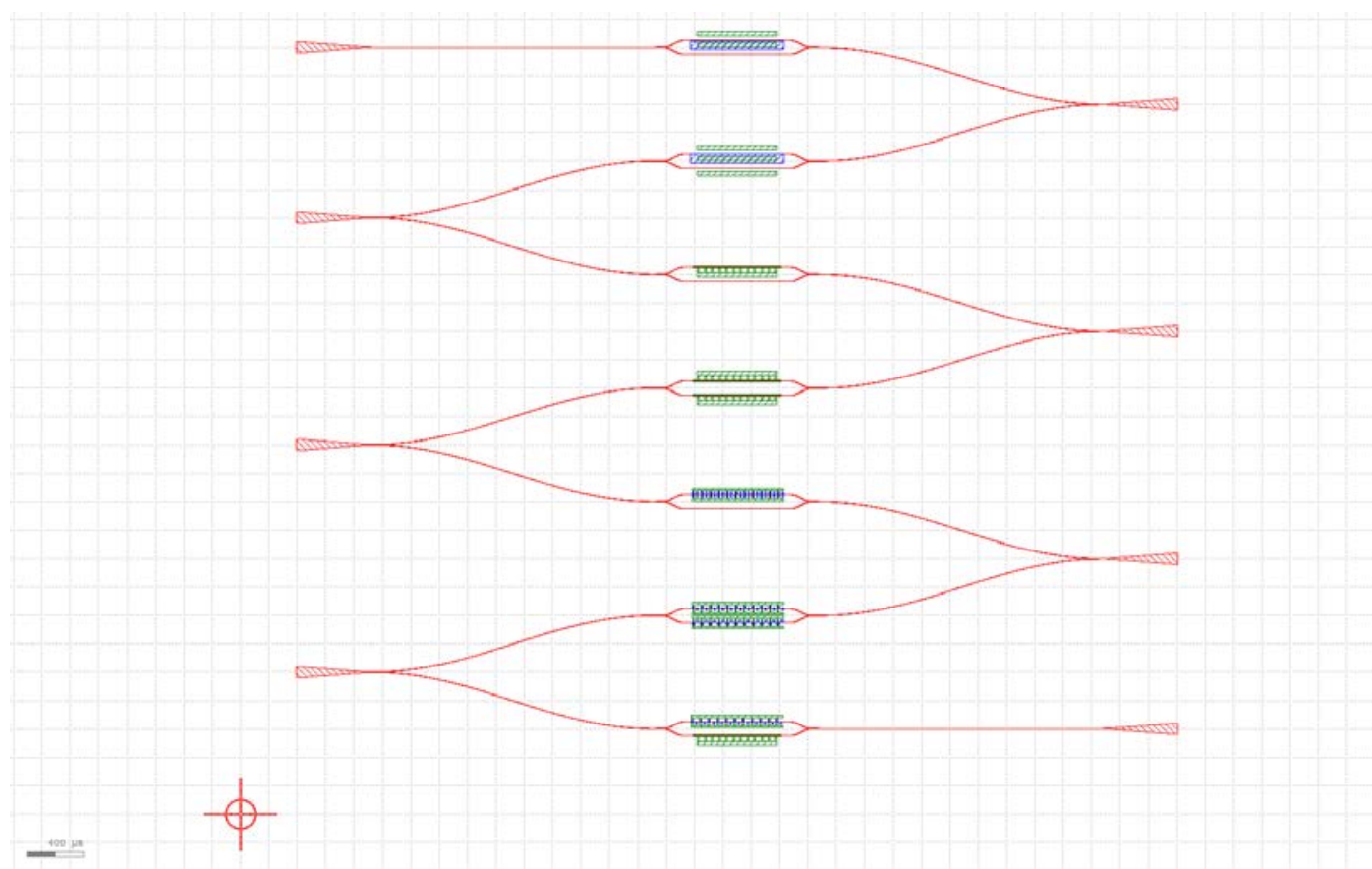

Figure 7: Unit Cell footprint.

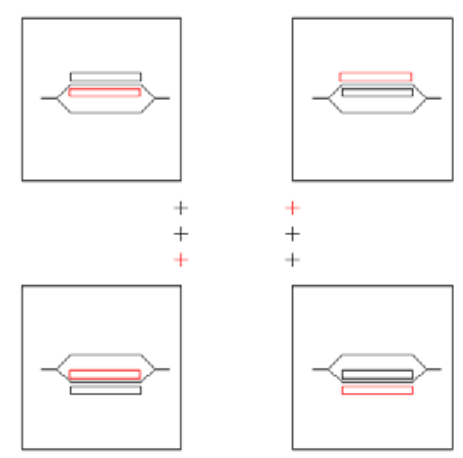

Figure 8: Symmetries on the wafer distribution.

9. Lithography for the waveguides pattern-The first mask to use is for the waveguides layer, this mask is designed to use negative photoresist. S1818 pos. photoresist must be used for this procedure. The coating must have a thickness not higher than $5 \mu \mathrm{m}$. The alignment between wafer and mask is not critical in this step. The exposure time it's estimated to be 90 seconds. Under or over exposure leads to change on effective index of the waveguide. The steps to obtain a $1.6 \mu \mathrm{m}$ coat and exposure procedure are provided.

10. Etch waveguides pattern and clean wafer- To etch the waveguides pattern, $\mathrm{XeF}_{2}$ dry etch must be used. 
11. Doping zones pattern P zones- After the cleaning process, for the doping zones we must use positive photoresist. Using the "doping” mask repeat the instructions on Step 9 and 10 adapting the values for the new photoresist.

12. Doping the P zones- For this step the doping furnace is used and procedure manual is followed to decide the doping level.

13. $\mathrm{N}$ zones doping pattern - As the design has a rotation symmetry, to pattern the N-zones regions in the wafer, mask is rotated by $180^{\circ}$ and the process steps 11 and 12 are repeated.

14. Deposit cladding layer / oxide

15. Pattern for metal contacts (+ res). To get the position of metal contacts, positive photoresist is used. Using photoresist S1818 and only changing the exposure time to get an underexposure to facilitate the lift-off process.

16. Etch oxide.

17. Deposit metal layer.

18. Pattern for metal contacts ( - res)

19. Etch metal

20. Place device wafer on the micromanipulator stage and use the CCD camera plus the EPIX software to capture images of MZI modulators. Examples are shown in figure 9.

21. Dice the wafer.

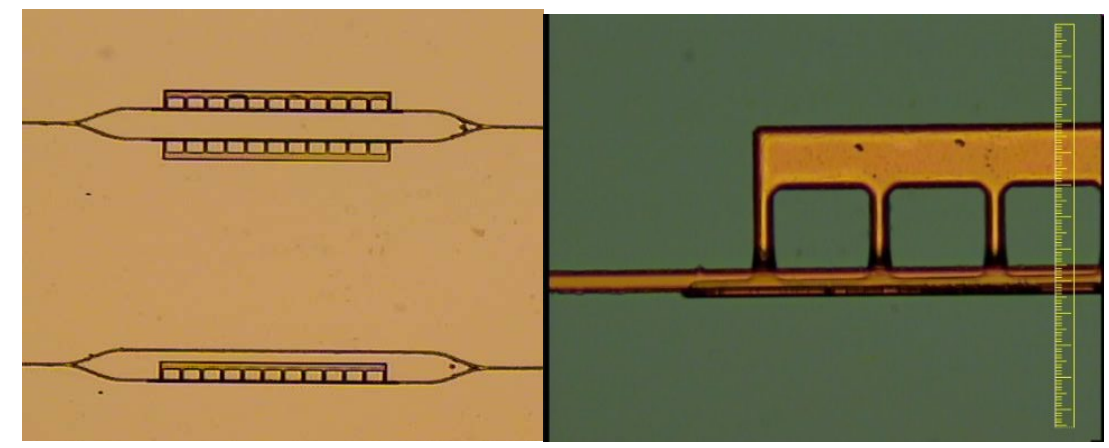

Figure 9: Wafer after wet metal etch under Micromanipulator.

For metal contact and heat pads nickel is sputtered. Nickel (Ni) etches isotropically in acidic solutions. The etch rate for Nickel in acidic bath is typically on the order of $10-15 \mathrm{~nm} / \mathrm{min}$ at $55^{\circ} \mathrm{C}$. The etch rate of $\mathrm{SiO}_{2}$ in acidic medium is considerably slower than Nickel, so the etch effectively stops when the nickel layer is removed and the $\mathrm{SiO}_{2}$ layer is exposed. However, it is not advised that one leave a wafer in the etchant for an extended period of time because of the undercutting that can take place. Therefore, the time for which the wafer remains in the etchant solution should be based on the thickness of the nickel thin film on the wafer. Typically, one assumes an approximate deviation of these parameters of $10 \%$. What this means is that one should add an additional $10 \%$ to the calculated time for which a wafer should remain in the etchant. The photoresist mask that was patterned in the photolithography step will also be unreactive during the etch.

\section{CHARACTERIZATION OF THERMO-OPTIC MZI}

After fabrication each group of student will test and characterize a MZI-modulator of their choice using the SMF input and output for light from a DFB laser at $1550 \mathrm{~nm}$ wavelength to a high-speed photodetector. We will discuss a typical individual project and experimental work by a group of students and comment on student's learning and course evaluations for silicon photonics course. 
To examine the figure of merits of a SOI modulator fabricated in the cleanroom many equipment such as: laser diode, high-speed detector, fiber scope, micromanipulator apparatus, power supply, multi-meters, function generator, oscilloscope are required. A test set like the one shown in figure 10 is used for this purpose.

Students are asked to use:

- cleaver to cleave the tip of a SMF

- fiber scope to examine cleanness of a fiber Ferro

- imaging microscope to examine the cleaved tip of a SMF

- optical power meter to examine the optical power of the laser diode

- plot optical power vs. drive current

- optical spectrum analyzer to examine the lasing wavelength and bandwidth of the laser diode

- setup shown in Fig.10 to do a Continuity test of the Ni-pads by connecting an ohm-meter across one of the Ni-pads on the SOI chip

- power supply and an ammeter to perform an V-I tests of the same Ni-pad on the SOI chip by connecting a in series

- Signal generator to modulate the index of refraction of MZI and try a range of frequencies. Students are asked to comment on any discrepancies and sources of error and include a few photos of various features on the tested MZI.

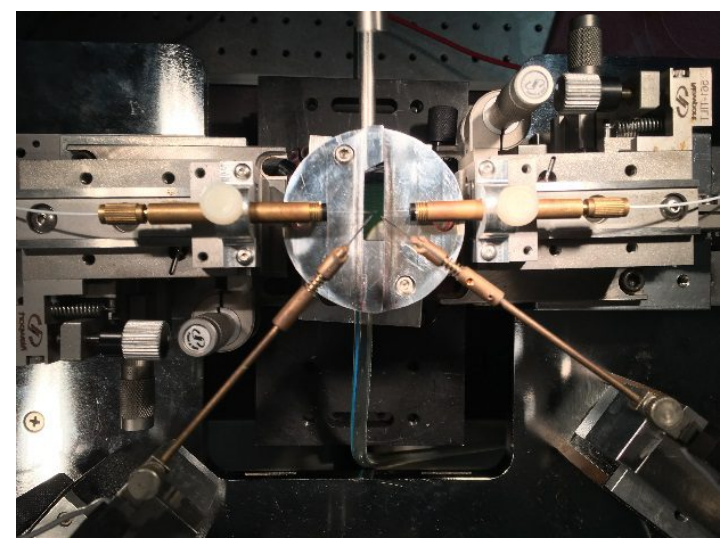

Figure10: Micromanipulator Apparatus for testing an SOI modulator.

\section{ACKNOWLEDGMENTS}

Author would like to thank all my graduate students who help with the fabrication and testing processes of this project and in particular: Jeongho Park, Pablo Nahuel Pelli, and Ehsan Ordouie.

\section{REFERENCES}

[1] Lukas Chrostowski and Michael Hochberg, "Silicon Photonics Design from Devices to Systems," Cambridge University Press, 2015.

[2] Robert A. Mayer, K. H. Jung, W. D. Lee, Dim-Lee Kwong, and Joe C. Campbell, "Thin-film thermo-o ptic $\mathrm{Ge}_{x} S i_{1-x}$ Mach-Zehnder interferometer," Opt. Lett. 17(24), 15(1992).

[3] R. A. Soref and B. R. Bennett, "Electro-optical effects in silicon," IEEE J. Quantum Electron. 23, 123129 (1987). 